\title{
Robotic thymectomy for myasthenia gravis
}

\author{
Marco Mammana, Giovanni M. Comacchio, Andrea Dell’Amore, Eleonora Faccioli, Elisa De Franceschi, \\ Sara Rossi, Federico Rea
}

Thoracic Surgery Unit, Department of Cardiac, Thoracic, Vascular Sciences and Public Health, University of Padua, Padua 35128, Italy.

Correspondence to: Dr. Marco Mammana, Thoracic Surgery Unit, Department of Cardiac, Thoracic, Vascular Sciences and Public Health, University of Padua, Via Giustiniani, 2, Padua 35128, Italy. E-mail: marcomammana87@gmail.com

How to cite this article: Mammana M, Comacchio GM, Dell'Amore A, Faccioli E, De Franceschi E, Rossi S, Rea F. Robotic thymectomy for myasthenia gravis. Mini-invasive Surg 2020;4:37. http://dx.doi.org/10.20517/2574-1225.2020.24

Received: 21 Feb 2020 First Decision: 20 Apr 2020 Revised: 21 Apr 2020 Accepted: 12 May 2020 Published: 18 Jun 2020

Science Editor: Piergiorgio Solli Copy Editor: Jing-Wen Zhang Production Editor: Jing Yu

\begin{abstract}
Thymectomy is an effective treatment option for the management of myasthenia gravis, as demonstrated by a recent multicenter randomized clinical trial. Complete removal of all thymic tissue, including ectopic foci, increases the chance of achieving a remission or a substantial improvement of the disease; therefore, extended transsternal thymectomy was long considered the procedure of choice. Over the years, several minimally invasive approaches have been proposed, with the aim to reduce perioperative morbidity and to improve aesthetics; however, concerns exist that through such approaches, it may not be possible to achieve a complete resection. Robotic thymectomy seems to overcome many of the limitations associated with other minimally invasive approaches. The available evidence suggests that robotic thymectomy for myasthenia gravis is a safe procedure, and that long-term neurological outcomes are satisfactory.
\end{abstract}

Keywords: Thymectomy, robot, myasthenia gravis

\section{INTRODUCTION}

Myasthenia gravis (MG) is a neuromuscular disease that manifests with fluctuating and fatigable weakness of different muscle groups. It occurs because of the production of autoantibodies directed against the components of the neuromuscular junction ${ }^{[1]}$. The medical management of MG includes the use of symptomatic therapy (anticholinesterase) and immunosuppressive treatment.

\footnotetext{
(@) (-)

(C) The Author(s) 2020. Open Access This article is licensed under a Creative Commons Attribution 4.0 International License (https://creativecommons.org/licenses/by/4.0/), which permits unrestricted use, sharing, adaptation, distribution and reproduction in any medium or format, for any purpose, even commercially, as long as you give appropriate credit to the original author(s) and the source, provide a link to the Creative Commons license, and indicate if changes were made.
}

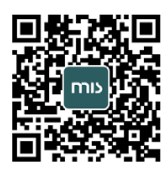


Blalock and colleagues ${ }^{[2]}$, in 1939 , were the first to report a dramatic improvement in symptoms following thymectomy in a patient affected by a cystic thymic tumor and MG. Since then, several other reports followed, highlighting a positive outcome of thymectomy in nonthymomatous $\mathrm{MG}^{[3]}$. However, in the absence of any formal evidence, the real benefit of this procedure remains in doubt.

Retrospective studies were analyzed by an in-depth review in $2000^{[4]}$; while the majority of reports demonstrated a favorable response from thymectomy in rates of disease remission or improvement, several methodological flaws precluded the investigators from drawing firm conclusions. A major breakthrough in the role of thymectomy for nonthymomatous MG was made only recently in 2016 by the Thymectomy Trial in Non-Thymomatous Myasthenia Gravis Patients Receiving Prednisone Therapy (MGTX) ${ }^{[5]}$. This large international, randomized, single-blind trial was conducted to determine whether extended transsternal thymectomy combined with a standardized prednisone protocol would be superior to prednisone alone after 3 years. A total of 126 patients from 36 institutions affected by generalized nonthymomatous MG, with strict inclusion criteria (age of 18 to 65 years, Myasthenia Gravis Foundation of America (MGFA) clinical class II to IV disease, positivity for acetylcholine-receptor (AChR) antibody, and disease duration less than 5 years) were randomized into the two treatment arms. The results from this study unequivocally demonstrated that thymectomy was beneficial with respect to clinical outcomes and requirements for prednisone therapy in patients affected by generalized nonthymomatous $\mathrm{MG}^{[6]}$.

Over the years, surgical approaches to thymectomy have evolved, with the aim of reducing surgical morbidity and of increasing the acceptance of such procedure for benign diseases, especially in young patients. Minimally invasive approaches include transcervical, videothoracoscopic (VATS), subxyphoid, and robot-assisted (RATS; robot-assisted thoracic surgery) thymectomy ${ }^{[7]}$. Various authors and metaanalyses have demonstrated that minimally invasive approaches to thymectomy are associated with better surgical outcomes and fewer surgical complications than the transsternal open approach, with no significant differences in MG complete remission rates ${ }^{[8]}$.

Currently, there is no definitive evidence in the literature that supports the use of one minimally invasive approach over the others; therefore, the decision is mostly based on the surgeon's preference. Factors that play a role in the choice of the surgical approach are perceived difficulty, ergonomics and the learning curve of the procedure, as well as the possibility of carrying out a thorough, extended thymectomy, which means the removal of the whole thymus with the surrounding fatty tissue of the neck and the mediastinum ${ }^{[9]}$. This is a capital concept in surgery for MG, as various authors have demonstrated that ectopic thymic foci are interspersed in the anterior mediastinal fat in up to $98 \%$ of patients, and that the removal of all thymic foci increases the probability of a complete remission of MG after surgery ${ }^{[10,11]}$.

At the Division of Thoracic Surgery of Padua University Hospital (Italy), starting from 2002, we developed a program of RATS thymectomy, and we currently adopt this approach for all patients who undergo thymectomy for nonthymomatous $\mathrm{MG}^{[12]}$. In this article, the rationale, indications, technique and outcomes of robotic thymectomy for MG are reviewed.

\section{RATIONALE FOR ROBOTIC THYMECTOMY}

The most widespread robotic system nowadays is the Da Vinci surgical system (Intuitive Surgical, Inc. Sunnyvale, CA, USA). This consists of a designed surgeon's console, a vision system, and a patient-side cart supporting the interactive robotic arms. The console is connected to the video system and the robotic cart, and it represents the interface between the surgeon and the robotic system. The surgeon sees the operative field through binoculars located in the upper part of the console and his/her fingers grasp the master controls below the display and moves the robotic arms. The system translates the three-dimensional 
movement of the hands and fingers into precise, identical, and real-time movements of surgical instruments inside the patient's chest.

Robotic thymectomy might be considered an evolution of the VATS approach. In fact, the high-resolution three-dimensional view of the operating field, attenuation of hand tremor and articulation of the robotic arms represent clear advantages of RATS over VATS thymectomy, especially in difficult to reach or narrow anatomical regions, such as the mediastinum. In the few studies where the RATS approach was compared with VATS, the investigators pointed out that the former approach is feasible and safe, and that it presents surgical advantages over the latter ${ }^{[13,14]}$. Moreover, Rückert et al. ${ }^{[13]}$ noted an improved outcome in myasthenic patients operated on by a robotic approach compared with those operated by VATS, which could have been due to the superior mediastinal dissection achieved with RATS. On the other hand, RATS thymectomy has some disadvantages. First, it is more expensive than VATS thymectomy, with most of the expense being due to the acquisition of the robotic system, its annual maintenance and the disposable materials. Second, there is a lack of tactile feedback that could increase the risk of damaging delicate anatomical structures. However, this seems to be widely compensated by the superior threedimensional view provided by the robotic console and the improved dexterity of robotic arms. Lastly, the operating surgeon is unscrubbed and placed away from the patient; therefore, in case of intraoperative complications requiring emergency conversion to sternotomy, another surgeon needs to stay sterile next to the patient ${ }^{[15-17]}$.

\section{PATIENT SELECTION AND PREOPERATIVE PREPARATION}

On the basis of current evidence, thymectomy is indicated for patients affected by generalized MG (grades II to IV, according to MGFA classification) and who are AChR antibody positive. No age limit exists; however, because it is an invasive procedure, the benefits of thymectomy have to be weighed against the risks of surgery, particularly in elderly patients. The chance of a complete remission of the disease decreases with age and with time from the onset of symptoms; therefore, there is general consensus that thymectomy should be offered early in the course of the disease of patients affected by $\mathrm{MG}^{[6]}$. Thymectomy may be offered also to MG patients without detectable levels of AChR antibodies; however, current guidelines do not support thymectomy in patients with MuSK, LRP4, or agrin antibodies ${ }^{[18]}$. Because of the long delay in onset of effect, thymectomy for MG is an elective operation; therefore, it should be proposed only to patients who are stable and deemed safe to undergo a procedure where postoperative pain and mechanical factors can limit respiratory function. In patients with thymomatous MG, surgery is indicated in any case to remove the tumor, regardless of the expected improvement in MG symptoms.

Preoperative workup includes contrast-enhanced computed tomography (CT), pulmonary function tests and blood gas analysis. The neurologist should evaluate all symptomatic patients to determine the need for intravenous immunoglobulin therapy or plasmapheresis in the immediate preoperative period.

\section{SURGICAL TECHNIQUE}

The surgical steps of robotic thymectomy are well described and there are only slight modifications in them across centers, as described elsewhere ${ }^{[19]}$. Both a right-sided and a left-sided approach are feasible, and, while every surgeon has a preferred approach (at our center this is the left-sided one), the procedure should be tailored on the patient's anatomy, and there should be no hesitation to add a contralateral incision if required. The main goal, in fact, should be to achieve a radical en-bloc resection of all thymic tissue, from one phrenic nerve to another, and from the inferior poles of thyroid gland to the diaphragm. Advantages of the left-sided approach include a usually larger distribution of the thymic gland and of the mediastinal fat to the left side and around the left phrenic nerve, accessibility to the aortopulmonary window, and a better visualization of the contralateral phrenic nerve, which is protected in its superior portion by the superior 

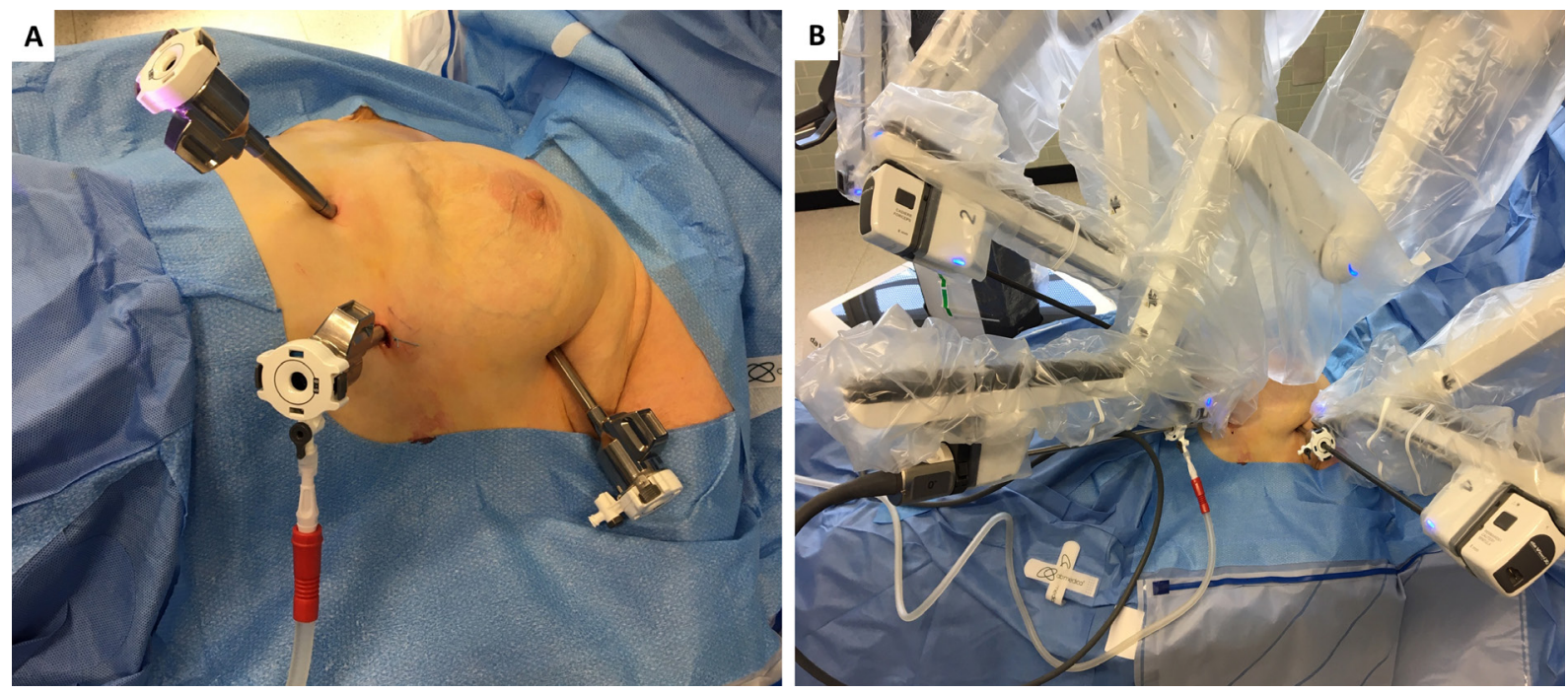

Figure 1. Port positioning and operative setup. A: the patient is positioned and draped. Ports are introduced on the fifth intercostal space on the midaxillary line, fifth intercostal space on the midclavicular line, and third intercostal space on the midaxillary line; B: the left arm is equipped with a grasping instrument (EndoWrist, Intuitive Surgical, Inc.), while the right arm has an Endo-dissector device (Intuitive Surgical, Inc.) with electric cautery function

vena cava. On the other hand, surgeons who prefer the right-sided approach like the larger space and the anatomical landmarks of the venous confluence.

The patient is under general anesthesia and single-lung ventilation. The operative side of the hemithorax is lifted $30^{\circ}$ from the supine position with the aid of a bean bag inserted under the patient's back. The field is prepared and draped for a conversion to median sternotomy or for addition of another port on the contralateral side [Figure 1A]. The procedure begins with insertion of the camera port through a 15-mm incision on the fifth intercostal space on the midaxillary line. The $\mathrm{CO}_{2}$ line is connected to the camera port and gas flow is regulated to an intrapleural target pressure of 6 to $10 \mathrm{mmHg}$; this helps in gaining space early into the procedure, particularly in the left-sided approach, where the camera port is very close to the heart apex. Two additional ports are placed under direct camera vision on the third intercostal space on the midaxillary line, and on the fifth intercostal space on the midclavicular line. Two arms of the da Vinci system are attached to the two access points and another arm is attached to the camera port. The left arm is equipped with an EndoWrist (Intuitive Surgical, Inc.) instrument; the right arm has an Endo-dissector device (Intuitive Surgical, Inc.) with electric cautery function [Figure 1B].

\section{Left-sided approach}

The dissection starts inferiorly at the level of the left cardiophrenic angle and continues along the anterior border of the phrenic nerve. All anterior mediastinal tissue, including fat, is isolated from the phrenic nerve. The left inferior horn of the thymus is then located and dissected from the pericardium. Subsequently, the thymic gland is separated from the retrosternal area until the right mediastinal pleura and the right inferior horn are found. At this point, the lower part of the thymus is moved upward, the left innominate vein is identified, and the dissection continues along the border of the innominate vein, up to the point where the thymic veins are identified, clipped, and divided. The dissection continues upward to the neck until the superior horns are identified and divided from the inferior portion of the thyroid gland. The thymus gland, anterior mediastinal, and neck fatty tissues are resected "en bloc", the medial port incision is slightly enlarged two fingerbreadths, and the specimen is then placed in an Endobag and removed. After hemostasis, a $28 \mathrm{~F}$ drain is inserted through the medial port, the lung is inflated, and the other wounds are closed. The patient is extubated in the operating room and then sent to the ward. 
Table 1. Main published series of robotic thymectomy for myasthenia gravis

\begin{tabular}{|c|c|c|c|c|c|c|}
\hline Ref. & Year & No. & Approach & Complete remission rate (\%) & Morbidity (\%) & Mortality (\%) \\
\hline Freeman et al. ${ }^{[20]}$ & 2011 & 75 & Left & 28 & 6.7 & 0 \\
\hline Ismail et al. ${ }^{[19]}$ & 2013 & 273 & Left & 57 & 1.6 & 0 \\
\hline Marulli et al. ${ }^{[21]}$ & 2013 & 100 & Left & 28.5 & 6.0 & 0 \\
\hline Keijzers et al. ${ }^{[22]}$ & 2015 & 125 & Right & 28.2 & 7.2 & 0 \\
\hline Kumar et al. ${ }^{[23]}$ & 2017 & 71 & Left & 38 & 7.0 & 0 \\
\hline
\end{tabular}

\section{Right-sided approach}

The mediastinal pleura is incised just anterior and medial to the right phrenic nerve, starting from the cardiophrenic angle and progressing upwards, and all anterior mediastinal tissue is separated from the nerve and the superior vena cava. The retrosternal parietal pleura is then opened medial and parallel to the right internal mammary vessels, and mediastinal tissue is dissected off the sternum anteriorly and the pericardium posteriorly, until the left brachiocephalic vein is identified. The thymic veins are identified, clipped, and dissected. The superior horns are then identified and divided from the thyroid gland. The left pleura is then opened and after the left phrenic nerve is identified, the dissection of the thymus is completed and the specimen is extracted as described above.

\section{OUTCOMES OF ROBOTIC THYMECTOMY}

The safety profile of RATS thymectomy seems excellent, with a morbidity rate ranging between $1.6 \%$ to $7.2 \%$ and no perioperative mortality in any of the studies [Table 1]. The most commonly reported complications include myasthenic crisis, bleeding and chylothorax ${ }^{[19-23]}$. In terms of postoperative results (blood loss, morbidity rate and length of hospital stay), several single-center case series have demonstrated better outcomes with RATS than with open thymectomy ${ }^{[24-26]}$. A multicenter study from the French database EPITHOR confirmed that patients undergoing thymectomy with minimally invasive procedures (mostly RATS) had fewer postoperative complications and a shorter hospital stay compared to patients operated on by sternotomy ${ }^{[27]}$. However, because of important disparities in baseline patients' characteristics, no firm conclusions about the superiority of one technique over the other could be drawn ${ }^{[27]}$. Finally, a recent systematic review compared postoperative outcomes after thymectomy by RATS or VATS, and found no significant difference in terms of morbidity, conversion to open and length of hospital stay ${ }^{[28]}$.

As far as neurological outcomes are concerned, in general, non-surgical factors that are believed to decrease the effectiveness of thymectomy in palliating symptoms of MG are the presence of thymoma (as compared with thymic hyperplasia), duration of symptoms longer than 1 year, and older age ${ }^{[29]}$. The completeness of removal of all thymic foci, on the other hand, is the single most important surgery-dependent variable that influences postoperative neurological outcomes ${ }^{[10,11]}$. Unfortunately, because of differences in surgical approaches and operative techniques, it is not always easy to determine the extent of removal of thymic tissue from retrospective studies. In an attempt to overcome this issue, the following definitions have been proposed: basic thymectomy includes the removal of the thymic gland without any surrounding fat; extended thymectomy includes removal of the thymus with surrounding fatty tissue of the neck and the mediastinum ${ }^{[30]}$; finally, the maximally extended thymectomy procedure, proposed by Jaretski, consists in removal of the thymus with all mediastinal fat, from the level of the upper poles of the thyroid gland to the diaphragm, with opening of both pleural cavities ${ }^{[10]}$. Clearly, the maximally extended procedure is recommended to achieve the highest remission rates. Zielinski and colleagues, in fact, have compared neurological outcomes of patients who underwent thymectomy according to 3 different techniques, demonstrating better complete remission rates in the group of patients treated by the most radical operative technique $^{[31]}$.

Following robotic thymectomy, all authors report satisfying complete remission rates, with values ranging from $28 \%$ to $57 \%{ }^{[19-23]}$. These results are in line with complete remission rates achieved by transsternal 
thymectomy, which range from $15.8 \%$ to $60 \%{ }^{[32]}$. Another neurological outcome measure is the proportion of patients experiencing an improvement of MG symptoms, as defined by the MGFA postintervention status classification, which ranges from $77 \%$ to $87.5 \%$ in robotic thymectomy series ${ }^{[20-23]}$. Again, these figures compare well with those reported after transsternal thymectomy, which leads to palliation rates (defined as symptom-free on medication or minimal symptoms on no medication) varying between $79 \%$ and $86 \%{ }^{[29]}$. Unfortunately, the limited number of patients, the variable inclusion criteria, the different measures used to define the neurological outcomes, as well as differences in operative techniques and surgical approaches, make it impossible to reliably compare neurological outcomes between transsternal and minimally invasive thymectomy, or thymectomy performed by different minimally invasive techniques (e.g., RATS, VATS and subxiphoid). To answer these questions, better designed, multicenter, randomized studies are needed.

\section{CONCLUSION}

The benefits of thymectomy for patients affected by nonthymomatous MG have now definitively been proven. RATS is a safe and effective minimally invasive approach to thymectomy, which provides satisfactory neurological outcomes and a reduced surgical morbidity compared to the transsternal approach. The lack of well-designed prospective studies makes it impossible to reliably compare surgical and particularly neurological outcomes between different surgical approaches.

\section{DECLARATIONS}

\section{Authors' contributions}

Conception and design of the study: Mammana M, Comacchio GM, Dell'Amore A, Rea F

Data analysis and interpretation: Mammana M, Comacchio G, Faccioli E, De Franceschi E, Rossi S

\section{Availability of data and materials}

Not applicable.

\section{Financial support and sponsorship}

None.

\section{Conflicts of interest}

All authors declared that there are no conflicts of interest.

\section{Ethical approval and consent to participate}

Not applicable.

\section{Consent for publication}

Not applicable.

\section{Copyright}

(c) The Author(s) 2020.

\section{REFERENCES}

1. Gwathmey KG, Burns TM. Myasthenia gravis. Semin Neurol 2015;35:327-39.

2. Blalock A, Mason MF, Morgan HJ, Riven SS. Myasthenia gravis and tumors of the thymic region: report of a case in which the tumor was removed. Ann Surg 1939;110:544-61.

3. Oosterhuis HJ. Observations of the natural history of myasthenia gravis and the effect of thymectomy. Ann N Y Acad Sci 1981;377:678-90.

4. Gronseth GS, Barohn RJ. Practice parameter: thymectomy for autoimmune myasthenia gravis (an evidence-based review): report of the Quality Standards Subcommittee of the American Academy of Neurology. Neurology 2000;55:7-15.

5. Wolfe GI, Kaminski HJ, Aban IB, Minisman G, Kuo HC, et al.; MGTX Study Group. Randomized Trial of Thymectomy in Myasthenia Gravis. N Engl J Med 2016;375:511-22. 
6. Sonett JR, Magee MJ, Gorenstein L. Thymectomy and myasthenia gravis: A history of surgical passion and scientific excellence. J Thorac Cardiovasc Surg 2017;154:306-9.

7. Magee MJ, Mack MJ. Surgical approaches to the thymus in patients with myasthenia gravis. Thorac Surg Clin 2009;19:83-9.

8. Qi K, Wang B, Wang B, Zhang LB, Chu XY. Video-assisted thoracoscopic surgery thymectomy versus open thymectomy in patients with myasthenia gravis: a meta-analysis. Acta Chir Belg 2016;116:282-8.

9. Zielinski M, Hauer L, Hauer J, Pankowski J, Nabialek T, et al. Comparison of complete remission rates after 5 year follow-up of three different techniques of thymectomy for myasthenia gravis. Eur J Cardiothorac Surg 2010;37:1137-43.

10. Jaretzki A, Wolff M. "Maximal" thymectomy for myasthenia gravis. Surgical anatomy and operative technique. J Thorac Cardiovasc Surg 1988;96:711-6.

11. Masaoka A, Nagaoka Y, Kotake Y. Distribution of thymic tissue at the anterior mediastinum. Current procedures in thymectomy. J Thorac Cardiovasc Surg 1975;70:747-54.

12. Rea F, Marulli G, Bortolotti L, Feltracco P, Zuin A, et al. Experience with the "da Vinci" robotic system for thymectomy in patients with myasthenia gravis: report of 33 cases. Ann Thorac Surg 2006;81:455-9.

13. Rückert JC, Swierzy M, Ismail M. Comparison of robotic and nonrobotic thoracoscopic thymectomy: a cohort study. J Thorac Cardiovasc Surg 2011;141:673-7.

14. Jun Y, Hao L, Demin L, Guohua D, Hua J, et al. Da Vinci robot-assisted system for thymectomy: experience of 55 patients in China. Int J Med Robot 2014;10:294-9.

15. Augustin F, Schmid T, Sieb M, Lucciarini P, Bodner J. Video-assisted thoracoscopic surgery versus robotic-assisted thoracoscopic surgery thymectomy. Ann Thorac Surg 2008;85:S768-71.

16. Melfi F, Fanucchi O, Davini F, Viti A, Lucchi M, et al. Ten-year experience of mediastinal robotic surgery in a single referral centre. Eur J Cardiothorac Surg 2012;41:847-51.

17. Marulli G, Rea F, Melfi F, Schmid TA, Ismail M, et al. Robot-aided thoracoscopic thymectomy for early-stage thymoma: a multicenter European study. J Thorac Cardiovasc Surg 2012;144:1125-30.

18. Sanders DB, Wolfe GI, Benatar M, Evoli A, Gilhus NE, et al. International consensus guidance for management of myasthenia gravis: executive summary. Neurology 2016;87:419-25.

19. Ismail M, Swierzy M, Rückert JC. State of the art of robotic thymectomy. World J Surg 2013;37:2740-6.

20. Freeman RK, Ascioti AJ, Van Woerkom JM, Vyverberg A, Robison RJ. Long-term follow-up after robotic thymectomy for nonthymomatous myasthenia gravis. Ann Thorac Surg 2011;92:1018-22; discussion 1022-3.

21. Marulli G, Schiavon M, Perissinotto E, Bugana A, Di Chiara F, et al. Surgical and neurologic outcomes after robotic thymectomy in 100 consecutive patients with myasthenia gravis. J Thorac Cardiovasc Surg 2013;145:730-5; discussion 735-6.

22. Keijzers M, de Baets M, Hochstenbag M, Abdul-Hamid M, Zur Hausen A, et al. Robotic thymectomy in patients with myasthenia gravis: neurological and surgical outcomes. Eur J Cardiothorac Surg 2015;48:40-5.

23. Kumar A, Goyal V, Asaf BB, Trikha A, Sood J, et al. Robotic thymectomy for myasthenia gravis with or without thymoma-surgical and neurological outcomes. Neurol India 2017;65:58-63.

24. Weksler B, Tavares J, Newhook TE, Greenleaf CE, Diehl JT. Robot-assisted thymectomy is superior to transsternal thymectomy. Surg Endosc 2012;26:261-6.

25. Ye B, Li W, Ge XX, Feng J, Ji CY, et al. Surgical treatment of early-stage thymomas: robot-assisted thoracoscopic surgery versus transsternal thymectomy. Surg Endosc 2014;28:122-6.

26. Seong YW, Kang CH, Choi JW, Kim HS, Jeon JH, et al. Early clinical outcomes of robot-assisted surgery for anterior mediastinal mass: its superiority over a conventional sternotomy approach evaluated by propensity score matching. Eur J Cardiothorac Surg 2014;45:e68-73.

27. Orsini B, Santelmo N, Pages PB, Baste JM, Dahan M, et al.; EPITHOR project (French Society of Thoracic and Cardiovascular surgery). Comparative study for surgical management of thymectomy for non-thymomatous myasthenia gravis from the French national database EPITHOR. Eur J Cardiothorac Surg 2016;50:418-22.

28. Fok M, Bashir M, Harky A, Sladden D, DiMartino M, et al. Video-assisted thoracoscopic versus robotic-assisted thoracoscopic thymectomy: systematic review and meta-analysis. innovations (Phila) 2017;12:259-64.

29. Daniel VC, Wright CD. Extended transsternal thymectomy. Thorac Surg Clin 2010;20:245-52

30. Masaoka A, Yamakawa Y, Niwa H, Fukai I, Kondo S, et al. Extended thymectomy for myasthenia gravis patients: a 20-year review. Ann Thorac Surg 1996;62:853-9.

31. Zielinski M, Hauer L, Hauer J, Pankowski J, Nabialek T, et al. Comparison of complete remission rates after 5 year follow-up of three different techniques of thymectomy for myasthenia gravis. Eur J Cardiothorac Surg 2010;37:1137-43.

32. Hess NR, Sarkaria IS, Pennathur A, Levy RM, Christie NA, et al. Minimally invasive versus open thymectomy: a systematic review of surgical techniques, patient demographics, and perioperative outcomes. Ann Cardiothorac Surg 2016;5:1-9. 